\title{
Dynamics of small particles in electromagnetic radiation fields: A numerical solution
}

\author{
Joonas Herranen ${ }^{\mathrm{a}}$, Johannes Markkanen ${ }^{\mathrm{a}}$, Karri Muinonen ${ }^{\mathrm{a}, \mathrm{b}}$ \\ ${ }^{a}$ Department of Physics, University of Helsinki, Finland \\ ${ }^{b}$ Finnish Geospatial Research Institute FGI, National Land Survey, Finland
}

\begin{abstract}
We establish a theoretical framework for solving the equations of motion for an arbitrarily shaped, inhomogeneous dust particle in the presence of radiation pressure. The repeated scattering problem involved is solved using a state-of-the-art volume integral equation-based $T$-matrix method. A Fortran implementation of the framework is used to solve the explicit time evolution of a homogeneous irregular sample geometry. The results are shown to be consistent with rigid body dynamics, between integrators, and comparable with predictions from an alignment efficiency potential map. Also, we demonstrate the explicit effect of single particle dynamics to observed polarization using the obtained orientational results.
\end{abstract}

\section{Introduction}

Electromagnetic scattering and the orientational state of the scatterer are coupled in a fundamental manner. Astronomically this is perhaps most importantly observed in the circular polarization of radiation scattered by dust, or dust polarization, which depends on nonrandom orientation of the dust. The connection of orientation and scattering is fundamental, still almost always the dynamical state of the scatterer is often "approximated away" from the problem. Yet, we are often interested in the full dynamical problem,

The effects of radiation pressure have been observed since the finding of cometary dust tails. Since then the dynamical effects of radiation were mainly of astronomical interest, as small weightless particles have the largest reaction to these minute forces. However, the extraterrestrial was made terrestrial when [1] showed that small particles can be trapped in laser beams. Since then, the newly multidisciplinary problem of radiation pressure has seen an accelerated development.

The observed polarization of the interstellar medium is due to scattering from asymmetrical, aligned dust particles. Alignment of interstellar dust particles has been under meticulous study for the last few decades, and it has been firmly established that the dominant alignment method in many situations is by radiative torques 2 . Study of radiative alignment methods coupled with an external magnetic field have been used, for example, in [3] to determine the galactic magnetic field from dust polar- ization measurements of the Planck mission.

In many applications, an efficient method to repeatedly solve the involving scattering problem is required. A long-lived problem has been to efficiently solve the underlying scattering problem for arbitrary shapes. Different discrete dipole scattering approaches [4, 5] are accurate, but repeated scattering problem solution is computationally expensive. On the other hand, the $T$-matrix method [6] is ideal for repeated solution of the scattering problem, but stable and efficient determination of the $T$-matrix for an arbitrary geometry has been a difficult problem. Previously, the discrete dipole approximation and approximative numerical methods have been applied to the problem [7, 8.

Current integral equation methods of scattering allow us to efficiently tackle the problem of scattering from arbitrary geometries. Using the electric current volume integral equation method [9], a numerically robust solution to scattering from strongly inhomogeneous particles is possible. Further still, the volume integral equation method can be applied to calculating the $T$-matrix of such particles [10. Radiation pressure in the form of radiative (optical in optical tweezer terms) forces and torques can be calculated efficiently using the $T$-matrix [11.

Recent developments of solving the scattering problem for arbitrary geometries are ideal for astronomical applications. Shape statistics are possible to be studied for different material properties, thus existing conclusions of the polarization inverse problem can be robustly verified 
and expanded upon with these methods.

In this work, we present a framework to study the time evolution of the rotation of an arbitrary particle, modeled both as a tetrahedral mesh and a spherical aggregate. The core program of the framework contains the definition and mass parameter determination of the geometry, the solution of the scattering problem and the numerical integration of the equations of motion. The formulation is based on current development of the volume integral equation methods of scattering and optical tweezer modeling. This allows the framework to be applied in the field of optical tweezers with few additions to the software.

In contrast with previous studies, we now present a numerically exact and fast realization of scattering dynamics. To the best of the authors' knowledge, this has not been presented in astronomical literature before. The methods used are powerful enough to solve the dynamics of arbitrary particles without any approximations or averaging to reduce the amount of scattering calculations. This makes the same framework usable in many different applications, including those described above, with minimal additions.

\section{Theoretical Framework}

In this section we introduce the necessary background for the development of a numerical solver of the rotational dynamics for an interstellar dust particle interacting with an electromagnetic field. Combining the following subsections, a framework for solving the equations of motion for a dust particle is obtained.

\subsection{Dynamics of a Rigid Body}

An interstellar dust particle is assumed to be an inhomogeneous rigid body which obeys Newtonian mechanics. Interstellar dust is composed of highly asymmetric particles, which in this work are modeled either as Gaussian random spheres (GRS) [12] or as spherical aggregates. In planetary science, similar geometries are successful models of dust particles [13. In the absence of a bank of interstellar dust specimen, these geometries are also the backbone of this work. The most important physical quantity of the particle is its moment of inertia tensor, the matrix form of which for a discretized body composed of mass points is defined as

$\mathbf{I}=\left(\begin{array}{ccc}\sum_{i} m_{i}\left(y_{i}^{2}+z_{i}^{2}\right) & -\sum_{i} m_{i} x_{i} y_{i} & -\sum_{i} m_{i} x_{i} z_{i} \\ -\sum_{i} m_{i} x_{i} y_{i} & \sum_{i} m_{i}\left(x_{i}^{2}+z_{i}^{2}\right) & -\sum_{i} m_{i} y_{i} z_{i} \\ -\sum_{i} m_{i} x_{i} z_{i} & -\sum_{i} m_{i} y_{i} z_{i} & \sum_{i} m_{i}\left(x_{i}^{2}+y_{i}^{2}\right)\end{array}\right)$, where the summations are done over all the mass points $m_{i}$ with coordinates $\left(x_{i}, y_{i}, z_{i}\right), i=1, \ldots, N$.

For an arbitrary tetrahedron, the method of standard tetrahedra [14] can be applied to calculate the moment of inertia tensor for each tetrahedral element. In the method, a tetrahedron is associated with a standard tetrahedron spanned by three isosceles right triangles with unit legs on the Cartesian axes, and its moment of inertia tensor is mapped back to the original tetrahedron. Standard formulae can be applied for spherical aggregates composed either of full spheres or concentric shells. Finally the parallel axis theorem can be used to find the total moment of inertia tensor.

As a real symmetric matrix the corresponding inertia matrix has an eigendecomposition $\mathbf{I}=\mathbf{Q I}_{\mathbf{p}} \mathbf{Q}^{\boldsymbol{\top}}$, where $\mathbf{I}_{\mathbf{p}}=\operatorname{diag}\left(I_{p, 1}, I_{2}, I_{3}\right)$ and $\mathbf{Q}$ is a rotation matrix. In the body frame, the rotational equations of motion simplify to Euler's equations,

$$
\mathbf{N}=\mathbf{I} \dot{\omega}+\omega \times(\mathbf{I} \omega)
$$

where $\mathbf{N}$ is the total external torque, $\omega$ is the angular velocity vector and $(\dot{)}$ is shorthand for a time derivative. Solving Euler's equations analytically is possible when proper constraints are introduced. For example, the angular velocity of torque-free rotation of an oblate spheroid is analytically solvable. An oblate spheroid has principal moments of inertia $I_{1}=I_{2}<I_{3}$. From this setup and an initial angular velocity $\omega=\left(\omega_{1}, 0, \omega_{3}\right)$ it is simple to show that the angular velocity in the principal coordinates will have form

$$
\omega(t)=\left(\omega_{1} \cos \Omega_{b} t, \omega_{1} \sin \Omega_{b} t, \omega_{3}\right) .
$$

Above, $\Omega_{b}=\frac{\left(I_{3}-I_{1} \omega_{3}\right)}{I_{3}}$ is a constant precession frequency of the angular velocity in the body frame.

For the orientation of the particle, unit quaternion approach simplifies calculations and dismisses gimbal lock problems [15]. Now the rotational dynamics of the particle are described by the equations of motion

$$
\begin{aligned}
\dot{q} & =\frac{1}{2} \omega q \\
\dot{\omega} & =\mathbf{I}^{-1}(\mathbf{N}-\omega \times(\mathbf{I} \omega)),
\end{aligned}
$$

where $q$ is the orientation unit quaternion of the particle.

In the quaternion formalism, rotation matrices are replaced by unit quaternions. The rotated vectors are replaced by their pure quaternion counterpart, whose real part is zero and the vector components give the three imaginary parts, $\omega=0+\mathrm{i} \omega_{x}+\mathrm{j} \omega_{y}+\mathrm{k} \omega_{z}$. In many algorithms, 


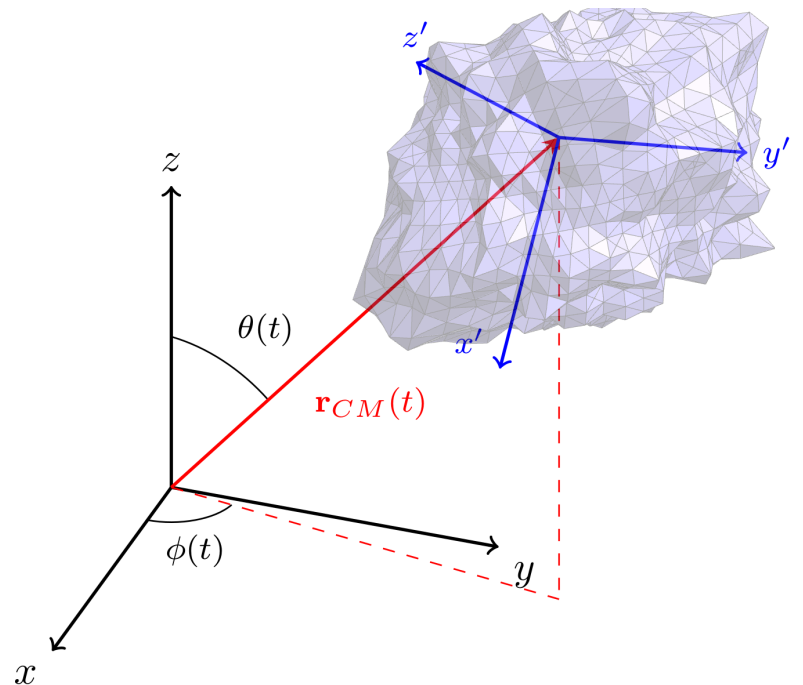

Figure 1: A mesh discretization of sample GRS particle in the laboratory frame $\{x\}_{\text {lab }}$ and a body frame $\left\{x^{\prime}\right\}_{\text {body }}$.

the time evolution of quaternions is clearer to implement than with rotation matrices. In addition, the numerical stability of the quaternion formalism is easily enforced by renormalizing the quaternion length to unity, which corresponds to enforcing the orthogonality of rotational matrix, a much less trivial task. The calculation of rotations itself is often, including in this work, done by switching back to matrix representation.

For a general description of the particle dynamics, the rotational and translational states with relation to the laboratory frame origin must be known at all times. The laboratory frame is most naturally fixed by any external direction, e.g. the direction of incident plane wave defining the $+z$-direction, and the initial positions. The rotational and translational states are given by the orientation and location of the principal body frame with respect to the laboratory frame. In the principal frame, the body frame axes align with the principal axes of the particle. This standard situation is described in Fig. 1. In an interstellar environment the rotational and translational dynamics can be treated separately, as we argue in the following subsections.

The rotation of a particle about its minor or major principal axis is known to be stable. For alignment of rotation to be possible, the particle naturally must be in a stable rotational state. Thus, a particle in a stable rotational state can be called internally aligned. Then the alignment with respect to some external direction can be accordingly called external alignment. In conclusion, a stable axis must be parallel or antiparallel to the angular

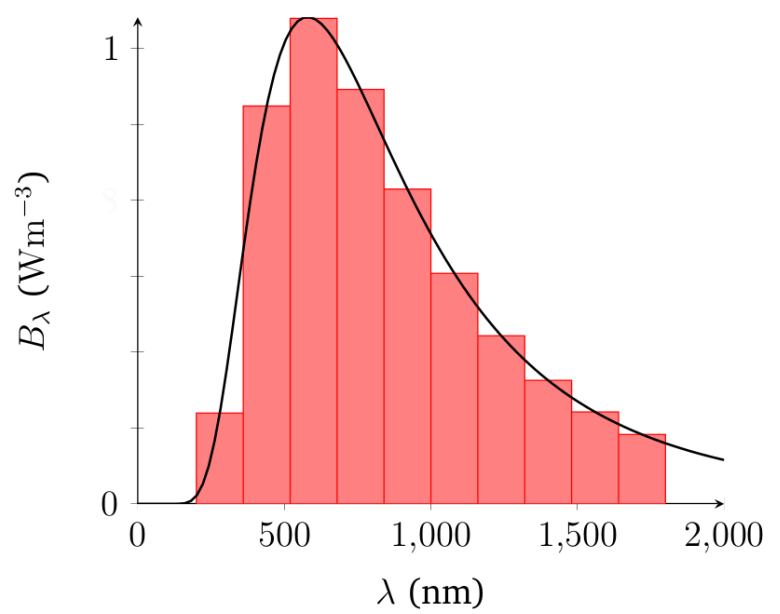

Figure 2: Discretization of a black body spectrum. Similar implementation is used throughout the framework, where the wavelength distribution always contains the wavelength $\lambda_{\max }$.

velocity and momentum with minimal precession.

\subsection{Electromagnetic Background}

The pressure effects of electromagnetic radiation were originally conceptualized in Kepler's observations of the tails of comets and formulated mathematically by [16]. The Maxwell equations form the basis of the problem, and are in the interstellar context most conveniently expressed in the time-harmonic plane wave form, without external sources, as

$$
\begin{aligned}
\mathbf{k} \cdot \mathbf{E}_{0} & =0, \\
\mathbf{k} \cdot \mathbf{H}_{0} & =0, \\
\mathbf{k} \times \mathbf{E}_{0} & =\omega_{f} \mu \mathbf{H}_{0}, \\
\mathbf{k} \times \mathbf{H}_{0} & =-\omega_{f} \varepsilon \mathbf{E}_{0},
\end{aligned}
$$

where $\mathbf{E}_{0}, \mathbf{H}_{0}$ are the amplitudes of plane waves of the form $\mathbf{E}=\mathbf{E}_{0} \exp \left(\mathrm{ik} \cdot \mathbf{x}-\mathrm{i} \omega_{f} t\right), \omega_{f}$ is the frequency of radiation, $\mathbf{k}$ the direction of propagation, and $\varepsilon, \mu$ the relative permittivity and permeability, respectively.

Realistic background radiation of the particles can be modeled as a black body spectrum, when the greatest contribution is of a single star. Such spectrum can be discretized as a piecewise constant distribution of intensities, as illustrated in Figure 2. The intensities can in turn be converted to corresponding electric field amplitudes at each wavelength.

A Lorentz force density, the force per unit volume, is

$$
\mathbf{f}=\rho \mathbf{E}+\mathbf{J} \times \mathbf{B},
$$


where $\rho$ is the charge of the volume element and $\mathbf{E}, \mathbf{J}$ and $\mathbf{B}$ are the electric field, electric current and the magnetic field intensity, respectively. By the Maxwell equations and vector calculus identities it can be expressed as

$$
\mathbf{f}=\nabla \cdot \mathbf{T}-\varepsilon_{0} \mu_{0} \frac{\partial}{\partial t} \mathbf{S}
$$

The $\mathbf{S}$-term, where $\mathbf{S}=\mathbf{E} \times \mathbf{H}$ is the Poynting vector, is the energy flux of the radiation fields, which has an average value zero in this context. The first term contains the Maxwell stress tensor with components

$$
T_{i j}=\varepsilon_{0}\left(E_{i} E_{j}-\frac{1}{2} \delta_{i j} E^{2}\right)+\frac{1}{\mu_{0}}\left(B_{i} B_{j}-\frac{1}{2} \delta_{i j} B^{2}\right) .
$$

Subtleties of the above expressions $(\sqrt{6}-(8)$ ) are discussed thoroughly by [17]. By integrating the force density to obtain the total force over a surface and using the divergence theorem, we obtain the total average mechanical force on the particle surface $S$,

$$
\mathbf{F}=\oint_{S} \mathrm{~T} \cdot \hat{\mathbf{n}} \mathrm{d} S
$$

The corresponding average torque due to EM radiation is, in a straightforward fashion,

$$
\mathbf{N}=\oint_{S} \mathbf{r} \times(\mathrm{T} \cdot \hat{\mathbf{n}}) \mathrm{d} S
$$

When applicable, the surface integral formulation of the force calculations are particularly useful in numerical calculations. Solving the scattering problem results in the knowledge of the total electromagnetic fields, and any method to solve the total fields can in principle be used to calculate the total radiative forces and torques.

\subsection{The solution of the scattering problem}

In the dynamical problem, we are interested in repeated solution of the scattering problem. When the particle can be approximated to be absolutely rigid, a single $T$-matrix completely describes the scattering properties of the particle.

The $T$-matrix for an arbitrary geometry is solved using the electric current volume integral equation (JVIE) formulation of scattering [9], which is an efficient approach for even strongly inhomogeneous scatterers. The JVIE formulation is then used to solve the $T$-matrix by linking the discretization basis function coefficients of JVIE by the method of moments with the vector spherical wave function (VSWF) expansion coefficients of the $T$-matrix method [10]. The above method can also be used to solve the $T$-matrix of an aggregate composed of identical particles whose $T$-matrices are known.

Using the $T$-matrix formulation the integrals $(9)$ and 10 can be solved analytically [11. The incident and scattered fields have VSWF expansions

$$
\begin{aligned}
E^{\mathrm{inc}} & =\sum_{n=1}^{\infty} \sum_{m=-n}^{n} a_{n m} \mathbf{M}_{n m}^{\mathrm{inc}}+b_{n m} \mathbf{N}_{n m}^{\mathrm{inc}} \\
E^{\mathrm{sca}} & =\sum_{n=1}^{\infty} \sum_{m=-n}^{n} p_{n m} \mathbf{M}_{n m}^{\mathrm{sca}}+q_{n m} \mathbf{N}_{n m}^{\mathrm{sca}}
\end{aligned}
$$

where $\mathbf{M}_{n m}^{\text {inc/sca }}, \mathbf{N}_{n m}^{\text {inc/sca }}$ are the incident and scattered VSWFs based on spherical Bessel functions and Hankel functions of the first kind, with expansion coefficients $a_{n m}, b_{n m}, p_{n m}$, and $q_{n m}$. There is some freedom in choosing the explicit form of the expansion, some standards can be found in e.g. [18, 19].

The expansion coefficients can be arranged into incident and scattered field vectors according to rule

$$
\begin{aligned}
\mathbf{a}= & \left(a_{1-1}, a_{10}, a_{11}, a_{2-2}, \ldots, a_{n_{\max }, n_{\max }-1}, a_{n_{\max }, n_{\max }}\right. \\
& \left.b_{1-1}, \ldots, b_{n_{\max }, n_{\max }}\right)^{\top}
\end{aligned}
$$

Then the vectors can be used in the $T$-matrix formulation of scattering simply as

$$
\mathbf{p}=\mathbf{T a}
$$

where $\mathbf{T}$ is the $T$-matrix of the scatterer. The truncation order $n_{\max }$ is usually set as in [20] to $n_{\max }=k r_{0}+3 \sqrt[3]{k r_{0}}$, where $k r_{0}$ is the size parameter of the particle. Size parameter is often defined as the product of the incident wavenumber and the volume equivalent radius, the radius of a sphere with equivalent volume. Thus the $T$-matrix size is solely determined by the relative size of the scatterer to incident wavelength, regardless of the complexity of the particle.

The integrals $(9)$ and $(10)$ can be computed most easily in the far-field limit. All momentum transfer is assumed to have happened in the scattering event. Thus the difference of the momentum and angular momentum between incident and scattered fields must be the transferred to the particle. The computation, using also the orthonormality and trigonometric properties of the spherical harmonics, 
results in sum formulae of the form [21]

$$
\begin{aligned}
F_{z}= & \frac{1}{k \sqrt{2 \zeta_{0}}} \sum_{n m} N_{F, n m}\left(a_{n m}^{*} \mathrm{i} b_{n, m}-p_{n m}^{*} \mathrm{i} q_{n, m}\right)+ \\
& M_{F, n m}\left(p_{n m}^{*} p_{n+1, m}-q_{n m}^{*} q_{n+1, m}-\right. \\
& \left.\left.a_{n m}^{*} a_{n+1, m}+b_{n m}^{*} b_{n+1, m}\right)\right), \\
N_{z}=- & \frac{1}{k^{2} \sqrt{2 \zeta_{0}}} \sum_{n m} N_{N, n m}\left(a_{n m}^{*} p_{n m}+b_{n m}^{*} q_{n m}-\right. \\
& \left.\left|p_{n m}\right|^{2}-\left|q_{n m}\right|^{2}\right),
\end{aligned}
$$

where $k$ is the wavenumber of the incident field and $\zeta_{0}$ is the impedance of the vacuum. The normalization constants $N_{F, n m}, M_{F, n m}$, and $N_{N, n m}$ depend on the explicit form of VSWF expansions. The constants can be determined by comparing the choice of expansions with the choices and the full formulae of [21]. Conveniently, the formulae of z-components can be used to determine the $\mathrm{x}$ - and $\mathrm{y}$-components when rotated using rotation operations for VSWFs [22]. Similar formulae can be determined directly for the $\mathrm{x}$ - and $\mathrm{y}$-components [11, but these are omitted for simplicity.

In plane wave illumination, the particle translation and rotation can be separated, as stated above. This is due to the fact that the particle illumination solely depends on the orientation of the particle with respect to the incident wave direction. This can be contrasted to the case of most optical tweezers, where the particle is illuminated by a shaped beam. Shaped beams require the use of VSWF translation formulae and addition of shape coefficients to the incident beam 23, 24].

The methods described are applicable in the situation, where a particle is spinning with nonrelativistic angular speeds. Purely relativistic effects, such as extreme Doppler shifts, can then be ignored. On the other hand, it is difficult to add external static fields directly to the existing scattering solution. These effects would make the $T$-matrices dependent on $\omega$, which provides considerable challenges to applying the method.

Fortunately, the extremely-low-frequency effects are negligible compared to the effects of scattering. For frequencies higher than zero, for example, the measurable effect of static magnetic field on a spinning dielectric is known as the Barnett effect 25]. Thus, effects that can be added to the $T$-matrix solution as extra terms, are possible additions to the method in the future. Other effects, that have no direct basis in electrodynamics, such gas bombardment, are also straightforward additions to the method.

\section{Numerical Methods}

A Fortran software for solving the equations of motion for an inhomogeneous particle, discretized by either tetrahedral meshing or by aggregation, has been developed by the authors. The software analyzes the mesh geometry, then calculates and diagonalizes the inertia matrix for the particle. Radiative torques and forces are calculated by an implementation of the JVIE $T$-matrix method, later abbreviated as T-VIE. The method makes it possible to calculate the forces and torques using formulae 14 in any particle orientation, if the primary fields are rotated in the calculations instead of the particle. This in turn makes a straightforward integration of the equations of motion possible without any approximations of the particle geometry other than what is limited by the mesh.

The problem setup is always in the laboratory frame, in which the incident radiation is toward the $+z$-direction. The equations of motion are solved in the principal axes frame (principal frame), and the scattering problem must be solved in some body frame. For simplicity, and as in this case there are no other reference directions in the laboratory frame, the body frame is chosen so that it equals the laboratory frame at $t=0$. Thus two rotation matrices, the time-dependent orientation matrix $\mathbf{R}$ and the diagonalization matrix $\mathbf{Q}$, are needed to navigate between the frames.

The matrix $\mathbf{R}(t)$ gives the current orientation of the scattering frame, where the mesh is in whatever orientation it was created, with respect to the laboratory frame. The general definition of such coordinate transformation matrix is

$$
\mathbf{R}=\left(\begin{array}{ccc}
\cos \left(x^{\prime}, x\right) & \cos \left(y^{\prime}, x\right) & \cos \left(z^{\prime}, x\right) \\
\cos \left(x^{\prime}, y\right) & \cos \left(y^{\prime}, y\right) & \cos \left(z^{\prime}, y\right) \\
\cos \left({ }^{\prime} x, z\right) & \cos \left(y^{\prime}, z\right) & \cos \left(z^{\prime}, z\right)
\end{array}\right)
$$

where $\left(x^{\prime}, x\right)$ represents the angle between the axis vector $x^{\prime}$ in $\left\{x_{\text {sca }}\right\}$ and the axis vector $x$ in $\left\{x_{\text {lab }}\right\}$. The matrix $\mathbf{Q}$ is given by the eigendecomposition of the inertia matrix $\mathbf{I}$, so

$$
\mathbf{Q}=\left(\mathbf{a}_{1}, \mathbf{a}_{2}, \mathbf{a}_{3}\right)
$$

where column vectors $\mathbf{a}_{1}-\mathbf{a}_{3}$ are the minor, intermediate and major (principal) axes of the mesh. The framework is summarized in Figure 3 .

Three integrators, a fourth-order Runge-Kutta method (RK) 26], a symplectic second-order Lie-Verlet integrator (VLV) [27], and a predictor-corrector direct multiplication method (PCDM) [28] are used to integrate the rotational 


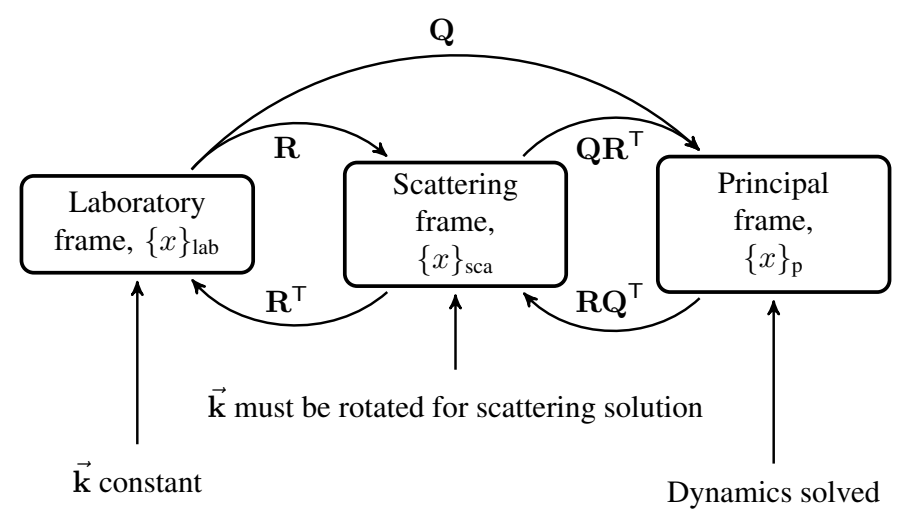

Figure 3: Diagram of the different coordinate transformations used and the significance of each frame in the integrator.

\begin{tabular}{|c|c|c|c|}
\hline$\frac{\text { Quantity }}{\text { Coordinates }}$ & $\overrightarrow{\mathrm{k}}$ & $\overrightarrow{\mathbf{N}}$ & $\mathbf{R}$ \\
\hline$\{x\}_{\text {lab }}$ & $\overrightarrow{\mathbf{k}}_{\mathrm{lab}}$ constant & $t=t+\Delta t$ & $\mathbf{R}(t)=\underset{\uparrow}{\mathbf{Q}^{\top}} \mathbf{R}_{\mathbf{p}}(t)$ \\
\hline$\{x\}_{\text {sca }}$ & $\overrightarrow{\mathbf{k}}_{\mathrm{sca}}=\mathbf{R}^{\top}(t) \overrightarrow{\mathbf{k}}_{\mathrm{lab}}$ & $\begin{array}{r}\overrightarrow{\mathbf{N}}_{\mathrm{sca}}(t) \\
\quad(2)\end{array}$ & (4) \\
\hline$\{x\}_{\mathrm{p}}$ & & $\overrightarrow{\mathbf{N}}_{\mathrm{p}}(t)=\mathbf{Q R}^{\boldsymbol{\top}}(t) \overrightarrow{\mathbf{N}}_{\mathrm{sca}}$ & $\mathbf{R}_{\mathrm{p}}(t)$ \\
\hline
\end{tabular}

Figure 4: Physical representation of the algorithm used in rotational integration. First, rotate the wave vector and solve torques using the $T$-matrix formulae; second, rotate the torque vector to the principal coordinates; third, update the equations of motion; and fourth, solve the new rotation matrix in laboratory coordinates.

motion of the test geometries. The orientational update steps, step (3) of Figure 4, are implemented as is from the references. Integrators are subject to constant development, but for an underconstrained system subject to nonHamiltonian interactions, only certain integration schemes are applicable. Such subtleties are considered e.g. by [29].

In the framework, orientation update steps are done in quaternion representation. For clarity, a matrix formulation of the algorithm is presented in the text. For forces, the integrators are demonstrated to reproduce expected behavior, as a plane wave should push the particles mostly along the direction of propagation. The translational motion is updated via the Runge-Kutta method. Physical integration framework is presented in Figure 4

To properly handle the stability of the rotational integration, adaptive time stepping must be used. A maximum angle of rotation $\theta_{\max }$ is used to determine the time step $\Delta t$ from the linear approximation so that

$$
\omega_{0}+\Delta \omega \leq \theta_{\max } / \Delta t
$$

As both the current angular velocity and the applying torque will affect the angle rotated during the time step, we approximate

$$
\Delta \omega / \Delta t_{\text {prev }}=\Delta \omega(\mathbf{N}(t), \omega(t)) / \Delta t_{\text {prev }}=\mathbf{I}^{-1}(\mathbf{N}-\omega \times(\mathbf{I} \omega)),
$$

where $\Delta t_{\text {prev }}$ is the previous time step and other quantities are the current values. If the old time step cannot satisfy the condition (17), the largest possible time step is chosen to replace $\Delta t_{\text {prev }}$.

\section{Results}

The particles considered in this work are solid and relatively small in the size parameter. For larger particles in size parameter, the single particle $T$-VIE method can be applied patiently for about up to size parameter 20. For even larger particles, aggregation described in [10] will be more efficient. The dimensions of the resulting $T$-matrices will naturally also depend on the size parameter, and thus have an effect on overall integration time.

First, we compare the force and torque calculations with respect to Mie solution and DDSCAT [30. All DDSCAT runs are made with minimal customization of parameters, namely, the discrete geometries are specified by their shape parameterization and the number of dipoles, and their electric properties by the index of refraction. The numbers of dipoles are chosen to produce self-convergent results in DDSCAT. Proper mesh discretization levels are chosen so that the maximum amount of tetrahedra in the smallest wavelengths are approximately same between tests. The comparative levels are named as crude, normal and fine in the figures.

Different geometries are used, with different size parameters $x=k a_{\text {eff }}$, and relative permittivity $2.842+\mathrm{i} 0.10521$. We focus on the dimensionless efficiency quantities, the force efficiency along the direction of radiation, or radiation pressure efficiency, $\mathbf{Q}_{p r}=\mathbf{F} /\left(\pi a_{\text {eff }} P\right)$, and the torque efficiency $\mathbf{Q}_{\Gamma}=\mathbf{N} k /\left(\pi a_{\text {eff }} P\right)$, where $a_{\text {eff }}$ is the volume equivalent radius, $P$ the power of the incident field and $k$ the wave number of the incident field. To be able to compare the torque efficiency results between $T$-VIE and DDSCAT, the particle is rotated so that the incident wave vector $\mathbf{k}$ and the major axis $\hat{\mathbf{a}}_{3}$ are parallel. In the discrete black body model of radiation, used in the full dynamical integrations, both efficiencies can be computed in a straightforward fashion for each wavelength.

In the force comparisons (in Figures 577) it is evident, that the error of the T-VIE solution is dependent on the quality of the mesh as size parameter varies (Figure 8). For realistic applications, for example when the incident 


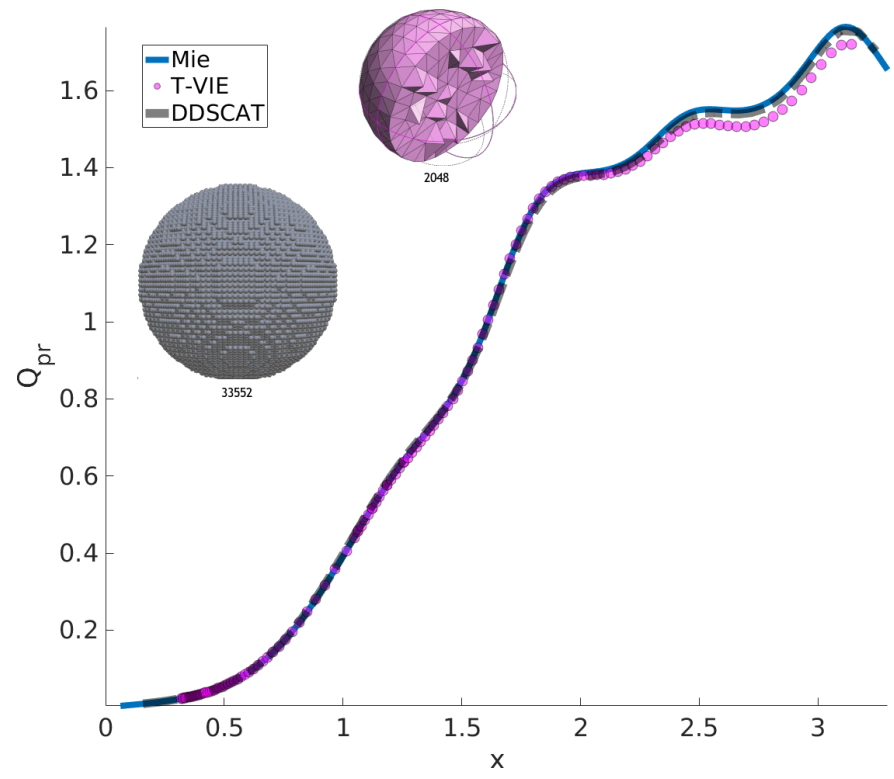

Figure 5: Comparison of force efficiencies $Q_{p r}$ along the direction of the incident radiation for a spherical particle in the Mie, DDSCAT, and $T$-VIE schemes as a function of the size parameter $x$. Tetrahedral mesh is scaled in this comparison, which results in the deviation in larger size parameters as less tetrahedra will fit in a single wavelength. The number of elements (tetrahedra or point dipoles) are below the geometry.

radiation field is of a black body, the shorter wavelengths become negligible quickly. This can be readily translated into concrete refinement rules when generating the geometries, so errors and computational efforts can be kept manageable. Later, we study the differences of dynamics of two GRS particles different in their level of discretization, with one geometry being the tetrahedral subdivision of the other. The difference between the efficiencies are illustrated in Figure 9 .

Then, we study the performance of the VLV integrator against the analytical solution (3) of the oblate spheroid. The spheroid is a tetrahedral discretization with $I_{p}=$ $(1.596,1.596,2.554) \mathrm{kg} \cdot \mathrm{m}^{2}$ with initial principal body frame angular velocity $\omega=(0.5,0,1) \frac{\mathrm{rad}}{\mathrm{s}}$. The problem is solved for $\theta_{\max }=0.1,0.01$, and 0.001 by calculating the first $10,000,100,000$, and 1,000,000 steps, respectively. The resulting angular velocity for $\theta_{\max }=0.1$ is plotted with respect to the analytical solution in Figure 10. The solutions are indistinguishable in the resolution of the figure. Also, the errors with respect to the analytical solution are plotted. The time stepping conditions $\theta_{\max }=0.01$ and 0.001 result in the same error, when simulated time is considered.

We demonstrate scattering dynamical integration with two uniform GRS particles, illustrated in Figure 1, dis-

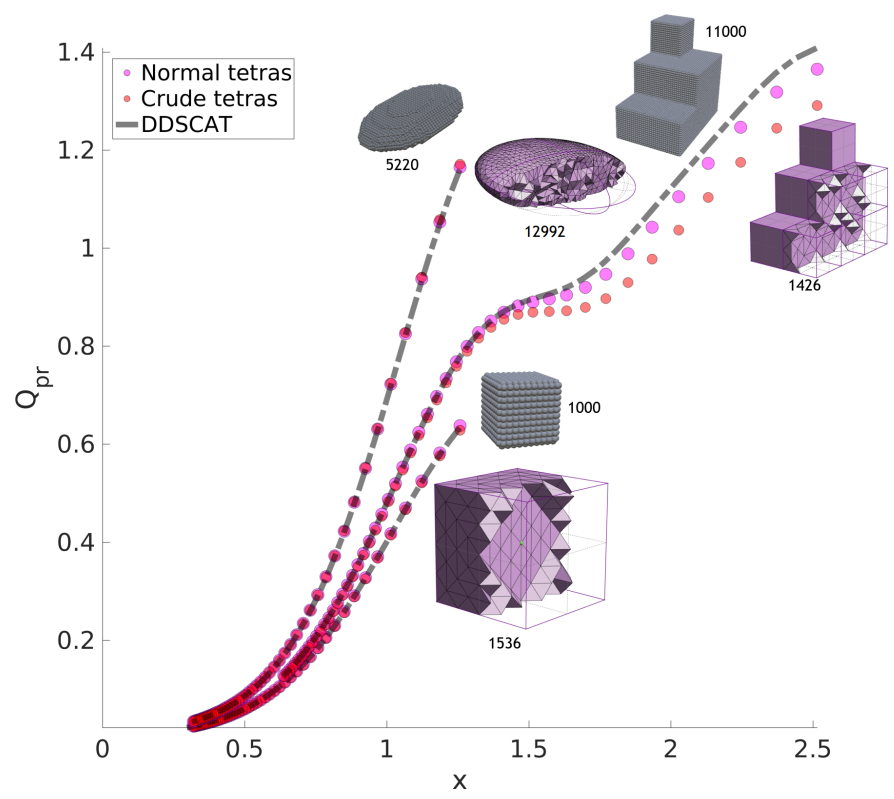

Figure 6: Force efficiency comparison for different geometries along the major principal axis. The normal tetrahedral meshes and DDA geometries are illustrated, with their number of elements. Cruder versions are composed of 1,624 (ellipsoid), 192 (cube), and 264 (multicube) tetrahedra.

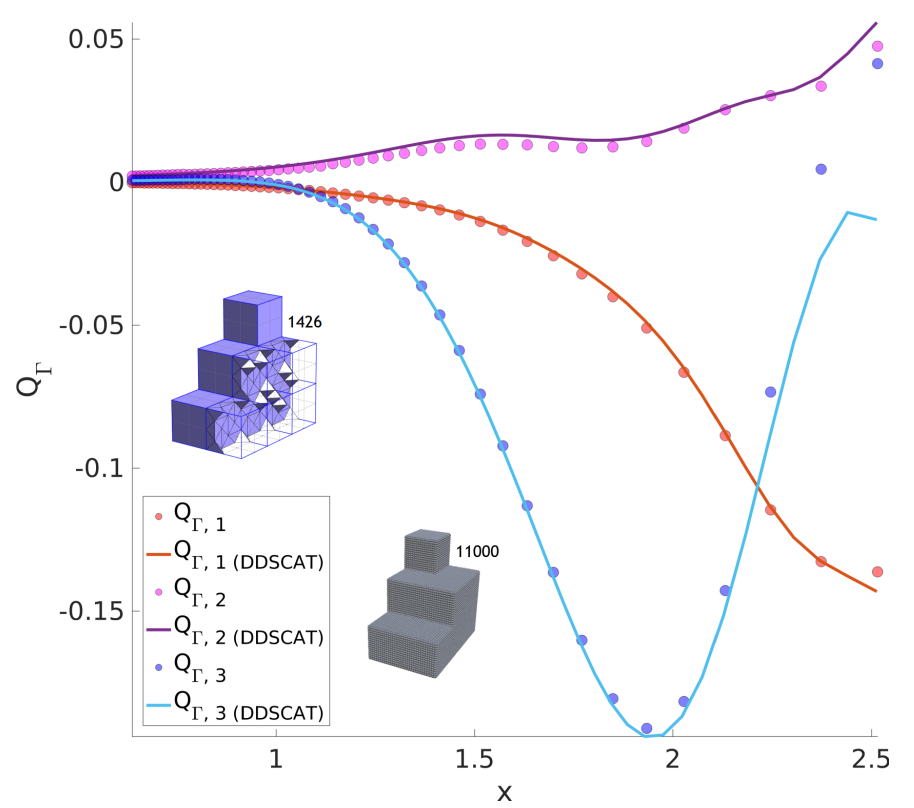

Figure 7: Torque efficiency components of an irregular block shape. In both images the effect of mesh quality is seen in larger size parameters as deviation. 


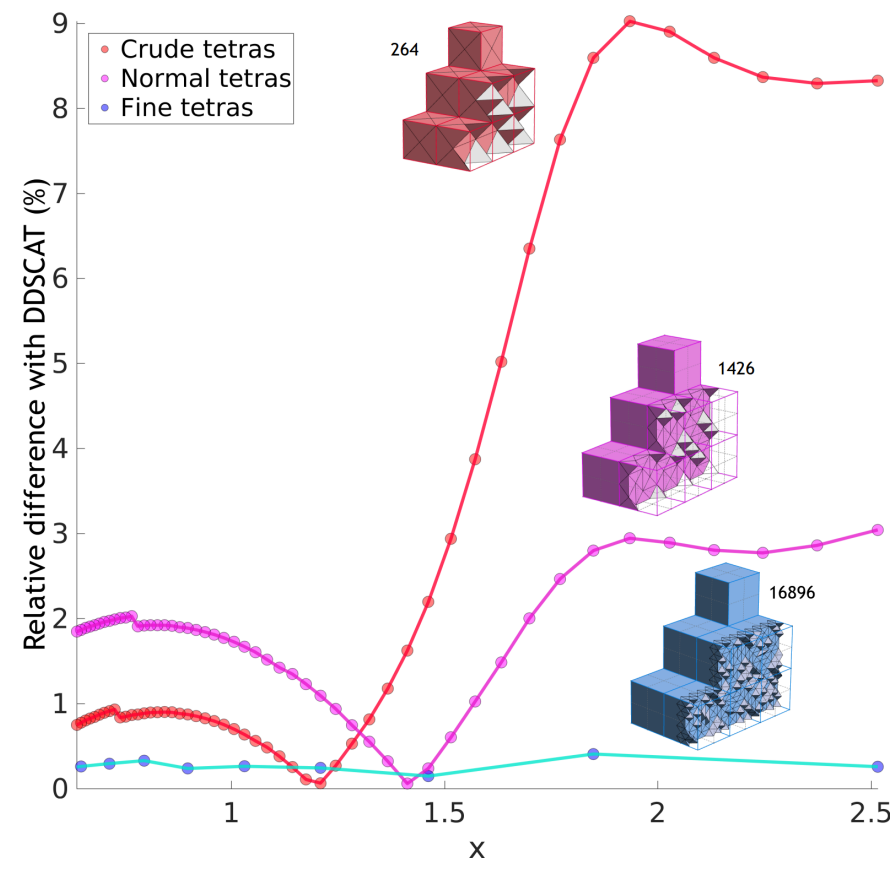

Figure 8: Relative difference of force efficiencies $\mid Q_{p r, \text { DDSCAT }}-$ $Q_{p r, T-\mathrm{VIE}} \mid / Q_{p r, \mathrm{DDSCAT}}$ between T-VIE and DDSCAT schemes as a function of size parameter for differently refined meshes.

Table 1: Initial orientation of the GRS particle principal axes

\begin{tabular}{ccc} 
Minor, $\mathbf{a}_{1}$ & Intermediate, $\mathbf{a}_{2}$ & Major, $\mathbf{a}_{3}$ \\
\hline 0.9174 & 0.3725 & 0.1396 \\
-0.3448 & 0.5695 & 0.7461 \\
0.1984 & -0.7327 & 0.6509 \\
\hline
\end{tabular}

cretized as a tetrahedral mesh with 6,049 and 48,392 elements. The particle is parametrized by volume equivalent radius $a_{\text {eff }}=0.2 \mu \mathrm{m}$, constant density $\rho=3,000 \frac{\mathrm{kg}}{\mathrm{m}^{3}}$, principal moments of inertia $I_{P}=(1.361,1.884,2.069)$. $10^{-30} \mathrm{~kg} \cdot \mathrm{m}^{2}$, and relative permittivity $2.842+\mathrm{i} 0.10521$. The initial directions of the principal axes are given in Table 1 The incident plane wave has an $n=10$-discretized black body spectrum of $T_{b b}=5,800 \mathrm{~K}$, with the total intensity corresponding to $E$-field amplitude of $1 \frac{\mathrm{V}}{\mathrm{m}}$. The wavelengths in the discrete spectrum are $0.500,0.680,0.860$, $1.040,1.220,1.400,1.580,1.760,1.940$, and $2.120 \mu \mathrm{m}$. The direction of propagation is along the $z$-axis in laboratory coordinates.

The effect of the choice of integrator, the level of discretization and the time stepping condition on the dynamics if shown in Figure 11, where the trajectories of the principal axis vectors and the angular velocity are plotted. The particle is initially at rest, and at the same initial orientation between runs. The simulations are run for either 100,000 or $1,000,000$ steps for $\theta_{\max }=0.01$ and 0.001 , respectively. The results are consistent between integrators and the time stepping conditions when considering the finer discretization.

Finally, we study the statistical behavior of the VLV and RK integrator schemes. Both the internal and external alignment of several hundred simulations are quantified. Internal alignment is measured from the final $2.5 \%$ steps of the run, after approximately 3,000 seconds of simulated time, as the average angle between the angular momentum and stable principal axes. The axis closer to internal alignment is also identified. This measure was chosen to count all precessing states as being stable. External alignment cannot be completely quantified, as there is only one external reference direction, the incident wave vector $\mathbf{k}$. Thus, the angle $\theta$ between the angular momentum vector and $\mathbf{k}$ is the only possible measure of external alignment.

Distributions of the rotational states are composed by choosing random initial orientations with same seeds for each different parameterizations, the discretization level, integrator, and time stepping condition. The resulting distribution of rotational states as histograms are collected in Figure 12. The major axis is seen to be the stably rotating one in almost all runs. From the raw data, it could be seen that not every run was the same, which is also reflected in the histogram, where perfect convergence of each run would result in exactly the same histograms.

To make sense of the dynamical results, an alignment efficiency map is included with the histograms. Alignment efficiency is defined here as the ratio of the torque efficiency along the major axis, $\mathbf{Q}_{\Gamma} \cdot \hat{\mathbf{a}}_{3}$. In the map, the quantity is computed in all possible orientations of the particle. The map is rotated such that the angle $\theta$ is equivalent with the earlier definition, and presented as the common Hammer projection of a sphere. The orientations, where most of the torque transferred to the particle is along the direction of the major axis, can be intuitively regarded as an stable orientation, given that the particle is internally aligned.

From Figure 12, it is seen that almost all particles are internally aligned at the end of the simulation. This is consistent with the results of 31. Comparing the values of $\theta$ in the regions, where alignment efficiency has an absolute value of at least $50 \%$, reveals a correlation with the histograms of external alignment. The most common alignment angle is in the range $120^{\circ}-160^{\circ}$, where in the alignment efficiency map there are two close "stability centers". Some external alignment is also observed around the range $3^{\circ}-80^{\circ}$. The level of alignment during the simulation con- 

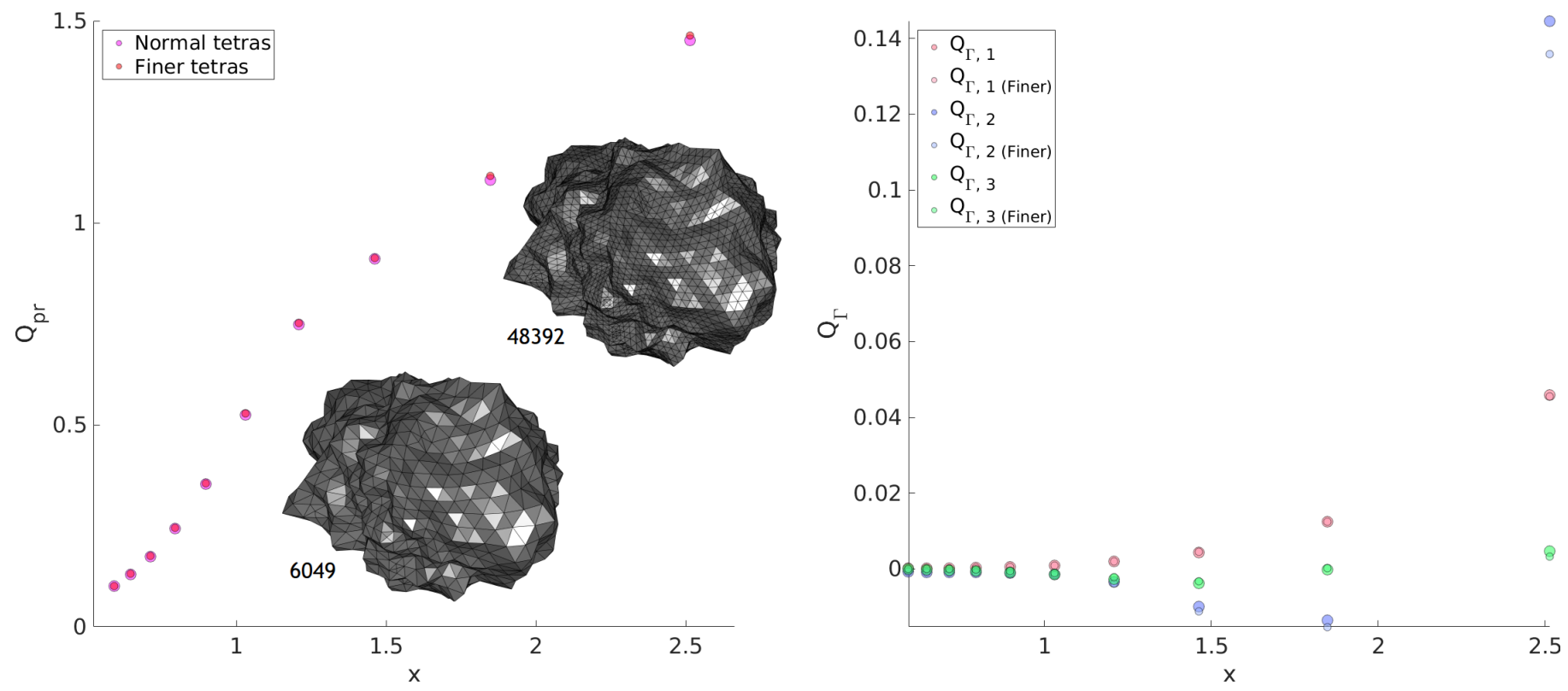

Figure 9: Force and torque efficiency comparisons for the two discretizations of the GRS particle. The higher discretization level results in similar correction to the efficiencies as in Figures 6 and 7
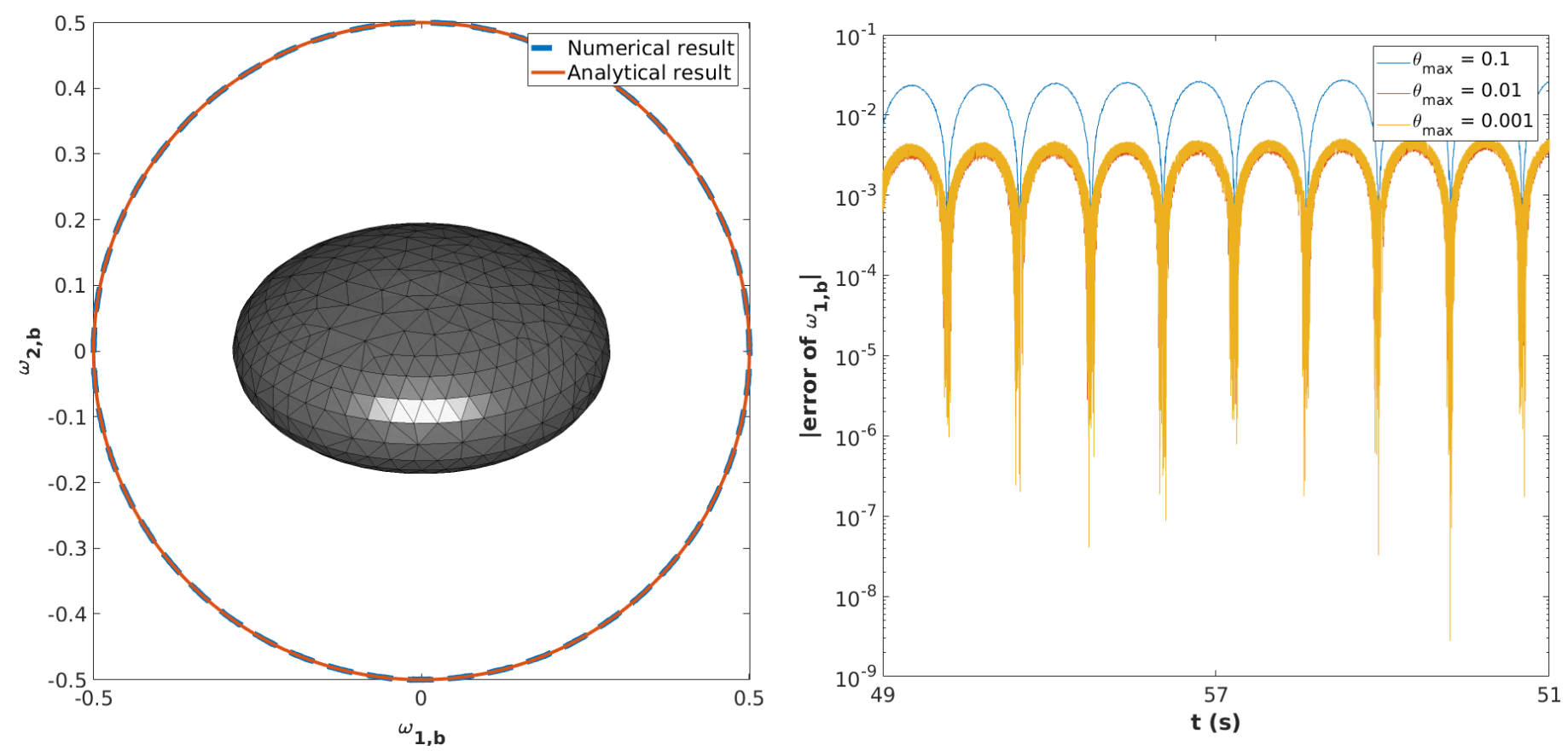

Figure 10: Analytical test of the VLV integrator for the oblate spheroid. The errors for cases $\theta_{\max }=0.01$ and 0.001 are very precisely at the same level, with the error for the case $\theta_{\max }=0.01$ visible only behind the oscillations. 

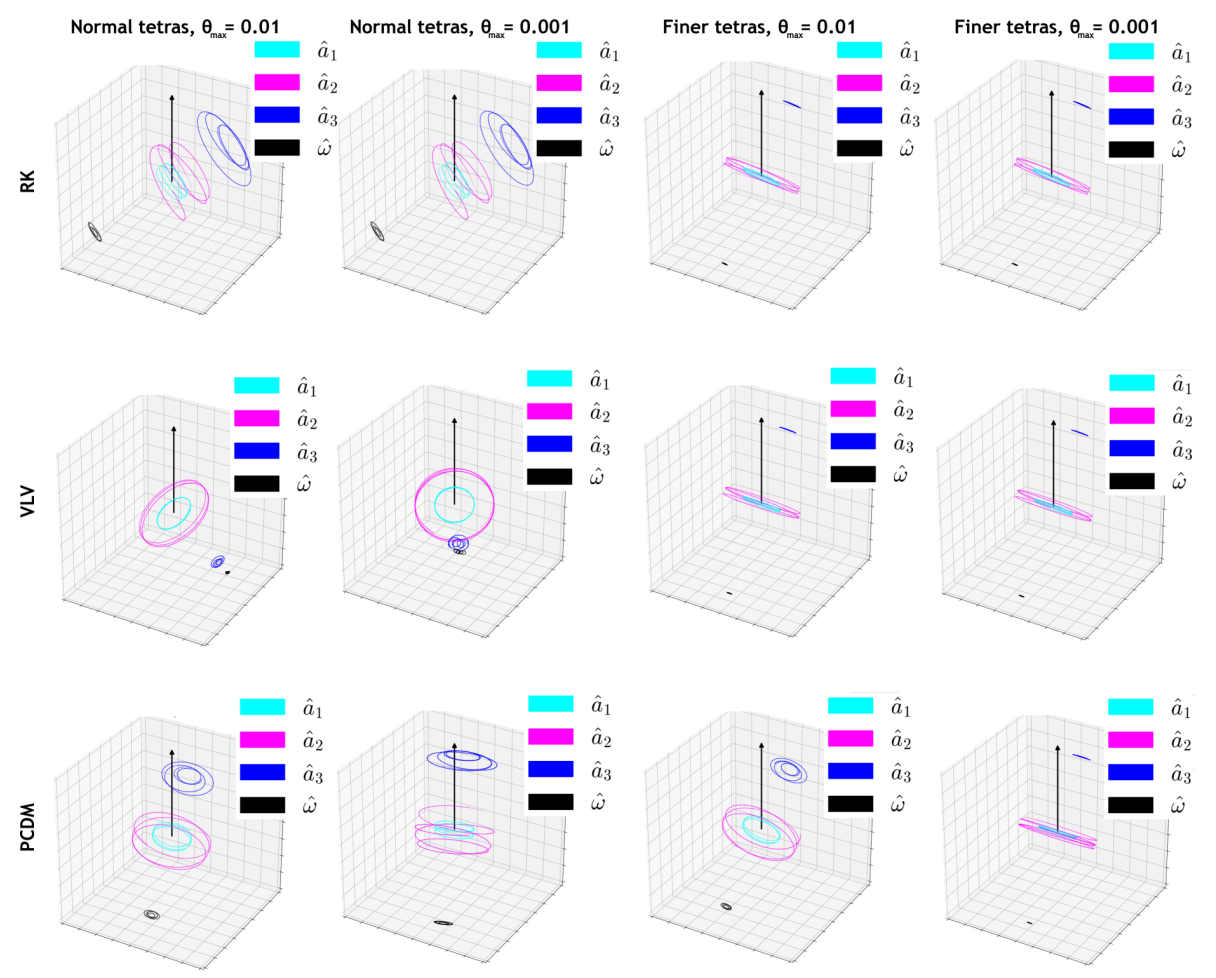

Figure 11: Integration comparison for the two different discretization levels of the GRS particle with two time stepping limits with 100000 steps for higher limit and 1,000,000 steps for lower limit.

sistently raises, which is expected, and the convergence of the most constrained initial setup is between 60 and $70 \%$, according to the raw data. The latter is not evident when studying the histograms, but the most self-consistent histogram column is of the most constrained case.

In Figure 13 , the well-known polarization effect is demonstrated by comparing the average Mueller matrix elements $S_{11}-S_{14}$. Averaging over random orientations results in strictly linear polarization, whereas oriented particle will have more diverse polarization. The rotational state, of which the Mueller matrix elements are calculated, is taken as the most constrained VLV integration from middle of the rightmost column in Figure 11. The scattering planes are defined by the incident wave vector and the average angular velocity direction. Two scattering planes, one containing the average angular velocity and other perpendicular to the first, are used as comparisons with the randomly oriented case. In both cases, significant circular polarizations are observed.

\section{Conclusions}

In this work, we have described a framework for studying the translational and rotational dynamics due to scattering for a single particle. We showed the framework to produce a stable rotational state of an arbitrary test particle in almost all cases, where the initial orientation was varied. Also, the alignment of the particle with stable rotational state was observed and shown to correlate with a measure of alignment potential, the alignment efficiency map. A perfect convergence of results between integrators was not observed in all cases. This implies, if trying to confine the error of the integrators is not desirable, a distribution of integration results should be the focus.

Further, we showed the polarization effect of oriented particles using the framework. The core of the framework can also be used in any application in which repeated solutions to a single scattering process is needed, for example to model optical tweezers.

The integrations considered relatively small angular velocities. This is in a sense a scaling issue, as the integration time step will ensure that completely same trajectories are achieved at different time scales in the torque-free case. The effect of optical torques will of course vary according to the angular velocity. As the choice of the magnitude of optical torques were determined arbitrarily, the dynamical effects were chosen to be studied starting at rest. Adding other torques than the optical torques to the system can have a considerable effect on the dynamics, changed by how the terms depend on the incident fields, the rotational 


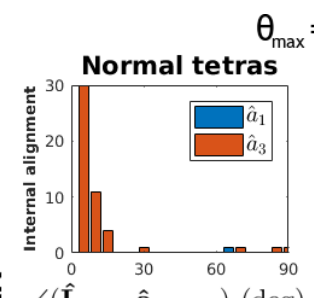

产
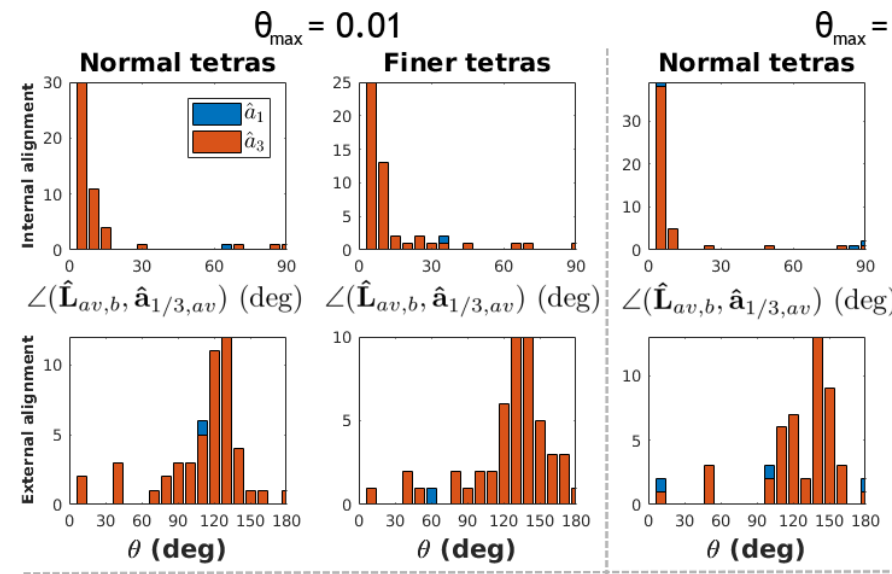

$\theta_{\max }=0.001$
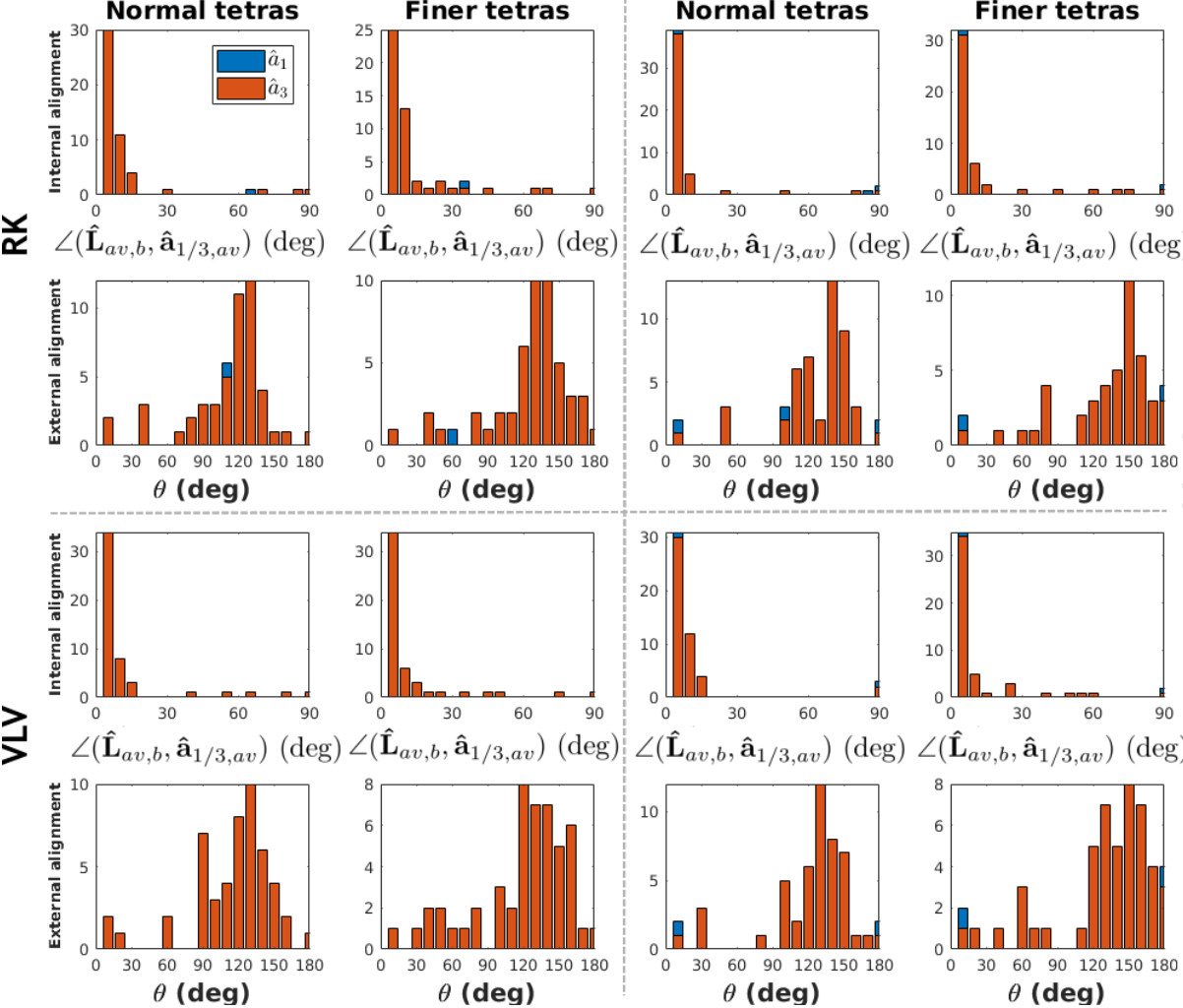

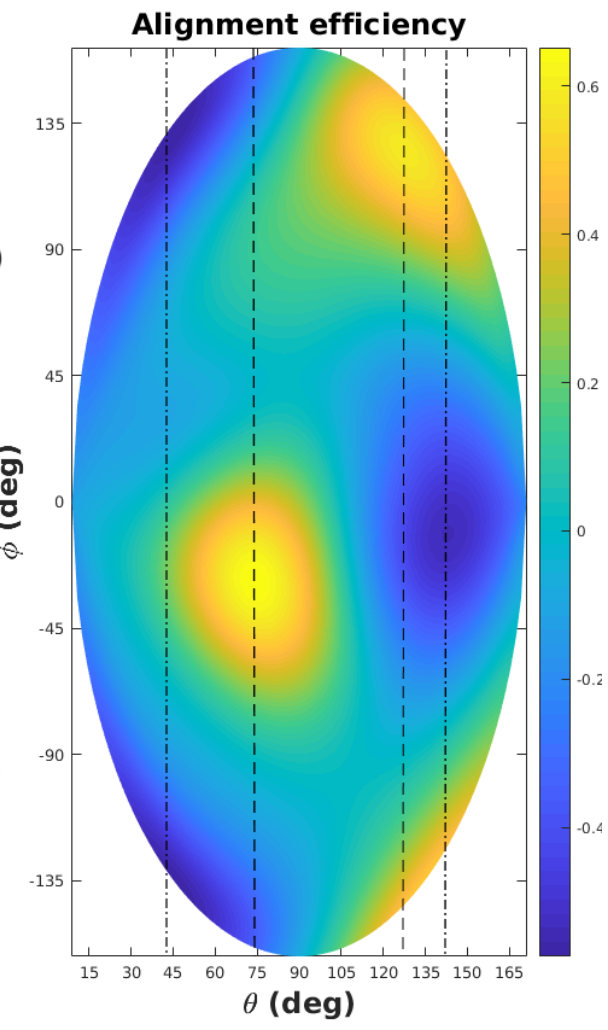

Figure 12: Probability distributions of alignment compared with the alignment efficiency map. Distributions contain both the internal and external alignment results for both RK and VLV integrators in the two different time stepping and discretization schemes.

state of the particle, or some statistical quantities such as collisions.

For future applications, different properties must be added to the framework. Addition of shaped beam VSWF coefficients will make the framework a cutting edge tool for studying optical tweezers. Similarly, coupling the translational and rotational motion will make it possible to study the dynamical separation of dust in dust clouds or cometary comae.

Some interesting applications, such as study of relativistic effects, external static magnetic fields, or electromagnetic emission, will challenge the limits of the $T$ matrix formalism itself. In some of these cases, a fallback into the underlying volume integral equation methods may be necessary.

The main benefit of the full dynamical integration is that many effects can be included as extra force and torque terms. For example, in the interstellar medium, particles are subject to collisions and external magnetic fields. Also, realistic particles go through dissipation of angular momentum. To summarize, if an effect can be considered an extra force or torque term, it can be added to the inte- gration scheme directly, whereas in potential energy approaches, for example, the same is not as intuitively clear to achieve.

\section{Acknowledgements}

The research has been supported, in part, by the ERC grant 320773 (SAEMPL) and the Academy of Finland grant 298137. We acknowledge the CSC for computing resources. The CSC is the Finnish IT center for science and is owned by the Ministry of Education.

\section{References}

[1] A. Ashkin, Acceleration and Trapping of Particles by Radiation Pressure, Phys. Rev. Lett. 24 (1970) 156-159. doi:10.1103/ PhysRevLett.24.156.

URL http://link. aps. org/doi/10.1103/PhysRevLett.24.156

[2] A. Lazarian, T. Hoang, Alignment of Dust by Radiative Torque: Recent Developments, ASPCS 449 (2011) 116-133.

[3] R. Adam, et al., Planck 2015 results. X. Diffuse component separation: Foreground maps, Astron. Astrophy. 594 (2016) A10. arXiv: 1502.01588

[4] B. Draine, J. Weingartner, Radiative Torques on Interstellar Grains: I. Superthermal Spinup, Astrophysical Journal 470 (1996) 551-565. doi:10.1086/177887 

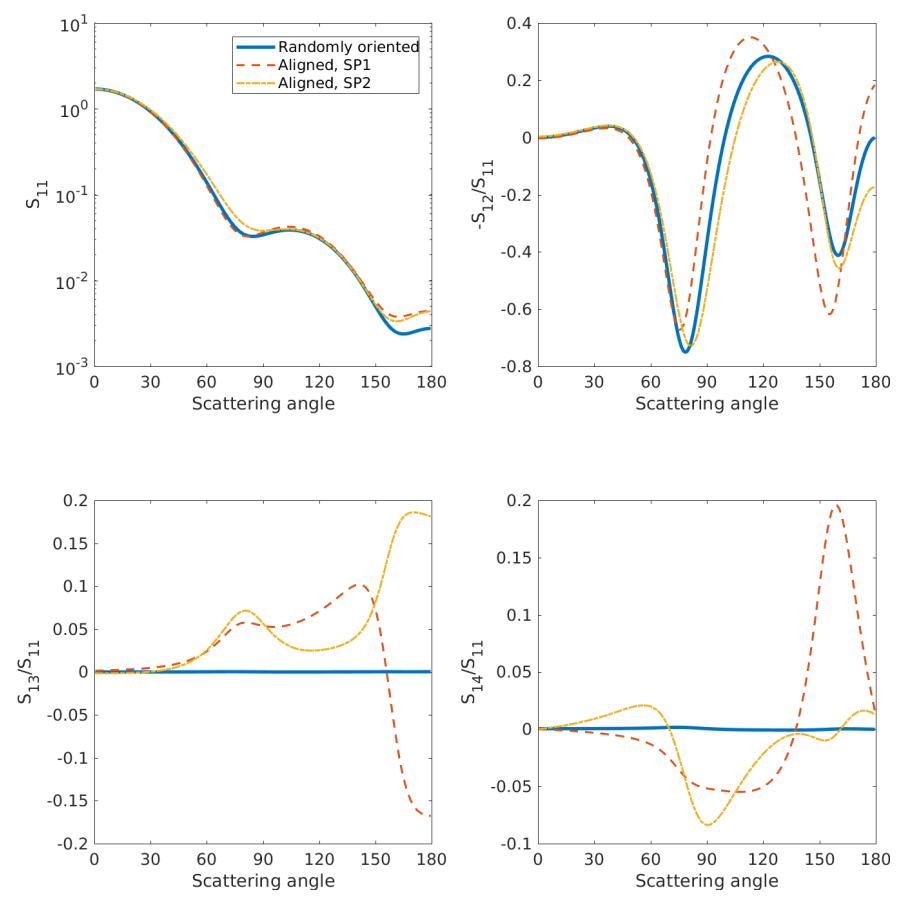

Figure 13: The Mueller matrix elements $S_{11}-S_{14}$. The aligned state is of the rightmost VLV case of Figure 11 Scattering plane 1 (SP1) is the plane containing the average of angular velocity of the particle. $\mathrm{SP} 2$ is a plane perpendicular to SP1.

[5] M. Yurkin, A. Hoekstra, The discrete-dipole-approximation code ADDA: Capabilities and known limitations, JQSRT 112 (13) (2011) 2234-2247.

[6] P. Waterman, Matrix formulation of electromagnetic scattering, Proceedings of the IEEE 53 (87) (1965) 805-812.

[7] D. Mackowski, Discrete dipole moment method for calculation of the T matrix for nonspherical particles, Journal of the Optical Society of America A 19 (2002) 881-893.

[8] V. Loke, T. Nieminen, N. Heckenberg, H. Rubinsztein-Dunlop, T-matrix calculation via discrete dipole approximation, point matching and exploiting symmetry, Journal of Quantitative Spectroscopy \& Radiative Transfer 110 (14) (2009) 1460-1471.

[9] J. Markkanen, P. Ylä-Oijala, A. Sihvola, Discretization of the volume integral equation formulation for extremely anisotropic materials, IEEE Transactions on Antennas and Propagation 60 (11) (2012) 5195-5202.

[10] J. Markkanen, A. Yuffa, Fast superposition T-matrix solution for clusters with arbitrary-shaped constituent particles, Journal of Quantitative Spectroscopy \& Radiative Transfer 189 (2017) 181-188.

[11] $\varnothing$. Farsund, B. Felderhof, Force, torque, and absorbed energy for a body of arbitrary shape and constitution in an electromagnetic radiation field Physica A: Statistical Mechanics and its Applications 227 (1-2) (1996) 108-130. doi:10.1016/ 0378-4371(96)00009-x.

URL https ://doi.org/10.1016\%2F0378-4371\%2896\%2900009-x

[12] K. Muinonen, T. Nousiainen, P. Fast, K. Lumme, J. Peltoniemi, Light scattering by gaussian random particles: Ray optics approximation, Journal of Quantitative Spectroscopy and Radiative Transfer 55 (5) (1996) 577 - 601, light Scattering by Non-Spherical Particles. doi:https://doi.org/10.1016/
URL http://www.sciencedirect.com/science/article/pii/ 0022407396000039

[13] L. Kolokolova, H. Kimura, Comet dust as a mixture of aggregates and solid particles: Model consistent with ground-based and space-mission results, Earth, Planets and Space 62 (1) (2010) 17-21. doi:10.5047/eps.2008.12.001 URL http://dx.doi.org/10.5047/eps.2008.12.001

[14] F. Tonon, Explicit Exact Formulas for the 3-D Tetrahedron Inertia Tensor in Terms of its Vertex Coordinates, Journal of Mathematics and Statistics 1 (1) (2004) 8-11.

[15] F. Zhao, B. Van Wachem, A novel Quaternion integration approach for describing the behaviour of non-spherical particles, Acta Mech 224 (12) (2013) 3091-3109.

[16] J. Maxwell, A Treatise on Electricity and Magnetism, Vol 2., Vol. 2, Cambridge University Press, 1873. doi:10.1017/ CB09780511709340

[17] T. A. Nieminen, N. du Preez-Wilkinson, A. B. Stilgoe, V. L. Loke, A. A. Bui, H. Rubinsztein-Dunlop, Optical tweezers: Theory and modelling Journal of Quantitative Spectroscopy and Radiative Transfer 146 (2014) 59-80. doi:10.1016/j.jqsrt. 2014.04 .003

URL https://doi.org/10.1016\%2Fj . jqsrt.2014.04.003

[18] J. Stratton, Electromagnetic Theory, Vol. 1, McGraw-Hill Company, 1941.

[19] J. D. Jackson, Classical Electrodynamics, Wiley, 1998, iSBN: 0-471-30932-X.

[20] C. Bohren, D. R. Huffman, Absorption and Scattering of Light by Small Particles, Wiley Science Paperback Series, 1998.

[21] J. Crichton, P. Marston, The measurable distinction between the spin and orbital angular momenta of electromagnetic radiation , Electron. J. Diff. Eqns., Conf. 4 (2000) 37-50.

[22] C. Choi, J. Ivanic, M. Gordon, K. Ruedenberg, Rapid and stable determination of rotation matrices between spherical harmonics by direct recursion, The Journal of Chemical Physics 111 (19) (1999) 8825-8831.

[23] J. Lock, G. Gouesbet, Rigorous justification of the localized approximation to the beam-shape coefficients in generalized Lorenz-Mie theory. I. On-axis beams, Journal of the Optical Society of America A 11 (9) (1994) 2503-2515.

[24] J. Lock, G. Gouesbet, Rigorous justification of the localized approximation to the beam-shape coefficients in generalized Lorenz-Mie theory. II. Off-axis beams , Journal of the Optical Society of America A 11 (9) (1994) 2516-2525.

[25] S. Barnett, Gyromagnetic and Electron-Inertia Effects, Rev.Mod.Phys 7 (1935) 129. doi:10.1103/RevModPhys.7.129

[26] W. H. Press, S. A. Teukolsky, W. T. Vetterling, B. P. Flannery, Numerical Recipes, Cambridge University Press, 2007.

[27] N. Bou-Rabee, J. Marsden, Hamilton-Pontryagin Integrators on Lie Groups Part I: Introduction and Structure-Preserving Properties, Found Comput Math 9 (2) (2009) 197-219.

[28] L. Seelen, J. Padding, J. Kuipers, Improved quaternion-based integration scheme for rigid body motion, Acta Mech 227 (12) (2016) 3381-3389.

[29] P. Pihajoki, Explicit methods in extended phase space for inseparable Hamiltonian problems, Celest Mech Dyn Astr 121 (3) (2015) 211-231.

[30] B. Draine, P. Flatau, Discrete-Dipole Approximation For Scattering Calculations, J. Opt. Soc. Am. A 11 (4) (1994) 14911499. 
[31] T. Hoang, A. Lazarian, Grain alignment induced by radiative torques: effects of internal relaxation of energy and complex radiation fields, ApJ 697 (2) (2009) 1316-1333. doi:10.1088/ 0004-637X/697/2/1316 